\title{
Olhares de género face à matemática: uma investigação no ensino obrigatório espanhol ${ }^{1}$
}

\author{
Júlio A. Gonzalez-Pienda \\ José Carlos Núñez \\ Paula Solano \\ Universidade de Oviedo, Espanha \\ Elza Helena da Silva \\ Universidade do Estado de Rio Grande do Norte \\ Pedro Rosário \\ Rosa Mourão \\ Universidade do Minho, Portugal \\ António Valle \\ Universidade de A Corunha, Espanha
}

\begin{abstract}
Resumo
O interesse pelo estudo das atitudes e o seu papel determinante na aprendizagem da matemática tem sido objecto de investigação há mais de 50 anos. Não obstante, actualmente, a investigação ainda se centra sobre as diferenças das atitudes das mulheres e dos homens, bem como sobre as causas de tais diferenças. No geral, os estudos realizados concluíram que, face à aprendizagem da matemática, as mulheres se percepcionam como menos competentes que os homens, embora estes estudos não sejam conclusivos. Outro dos dados emergentes sugere que, à medida que o estudante progride na escolaridade obrigatória, a atitude face à matemática vai sendo mais negativa. O presente trabalho aporta mais alguns dados sobre as diferenças nas atitudes face à matemática em função do género e do ano de escolaridade no contexto educativo espanhol.
\end{abstract}

Palavras-chave: atitude face à matemática; género; sucesso escolar

\begin{abstract}
Looking at Mathematics through gender: a study in Spanish compulsory education. Studying attitudes and their important role in learning Mathematics has been one of the concerns of research over the last fifty years. Nowadays research still deals with attitudes, but a new focus of interest comes to light: studying the differences between male and female attitudes and the reasons for that. Some existing studies state that females perceive themselves as less competent than males do in learning Mathematics but those studies are still not conclusive. Data also suggest that over school years students' attitudes grow more and more negative. The present study highlights the differences in attitudes towards Mathematics according to gender and school year in the Spanish educational context.
\end{abstract}

Keywords: attitudes towards mathematics; gender; school achievement

$\mathrm{O}$

sucesso na matemática constitui um desafio para a maioria dos sistemas educativos, não só porque a matemática é considerada como uma das disciplinas fundamentais do currículo escolar, mas também pela sua contribuição estruturante no desenvolvimento do conhecimento cognitivo e pelo carácter instrumental da maioria das aprendizagens matemáticas na vida adulta (Furner, Yahya, \& Duffy, 2005). Assumida esta importância, existe uma preocupação crescente nas sociedades modernas ocidentais pelo facto de uma parte importante dos alunos, e também da população em geral, exibir dificuldades evidentes em compreender e utilizar os conhecimentos matemáticos. Este cenário educativo, não exclusivo de nenhum sistema escolar em particular, não deixa de ser paradoxal. Por um lado, a matemática apresenta-se como um indiscutível conhecimento transversal, imprescindível nas sociedades modernas com um desenvolvimento tecnológico sem precedentes, mas, por outro lado, como por exemplo a realidade do sistema educativo castelhano, evidencia-a como 
um dos conhecimentos mais inacessíveis para muitos alunos (González-Pienda et al., 2002).

Na área da matemática concentram-se um grande número de dificuldades e fracassos escolares (Bishop, 2000; GonzálezPienda \& Alvarez, 1998). Para além disso, os erros e o baixo rendimento nesta matéria não afectam somente os alunos menos capacitados, já que muitos estudantes que exibem competência e alto rendimento noutras matérias escolares, obtêm resultados baixos ou negativos na matemática (González-Pienda et al., 2002; Núñez et al., 2005). A literatura, mas também a prática de ensino, referem que diversos alunos percebem a matemática como um conhecimento intrinsecamente complexo que gera sentimentos de ansiedade e intranquilidade, constituindo uma das causas mais frequentes de frustrações e atitudes negativas face à escola (Koehler \& Grouws, 1992).

Nos últimos anos, constatou-se um aumento de investigações relacionando a dimensão afectiva do indivíduo (crenças, atitudes e emoções) e o ensino/aprendizagem da matemática (Leder, 1992; McLeod, 1992; Utsimi \& Mendes, 2000). O domínio afectivo está a adquirir muito protagonismo neste campo sustentado na hipótese de que as atitudes, as crenças e as emoções influenciam quer o sucesso, quer o baixo rendimento e fracasso na aprendizagem da matemática. McLeod (1992) e Koehler e Grouws (1992) defendem, com dados das suas investigações, o papel prioritário da dimensão afectiva no ensino da matemática. São inquestionáveis os insucessos escolares nos mais variados níveis instrutivos alocados à matemática. Entre as diversas variáveis que influenciam esse insucesso encontram-se as atitudes negativas dos alunos face a esta área de estudo. Perante esta perspectiva, torna-se necessário encontrar respostas satisfatórias para esta questão (Kloosterman, Tassel, Ponniah, \& Essex, 2001). Watt (2000), por exemplo, realizou um estudo que tinha como principal objectivo conhecer a relação entre as atitudes e o rendimento académico na área da matemática, investigando de que modo esta se encontra influenciada pelos anos de escolaridade dos indivíduos. Os seus resultados foram consistentes com estudos anteriores, assinalando uma mudança de atitudes face à matemática ao longo da escolaridade; isto é, à medida que se avança na escolaridade observam-se atitudes mais negativas face à aprendizagem dos conhecimentos matemáticos, assim como uma tendência para a ascendência masculina neste domínio (Núñez et al., 2005).

Idênticos resultados foram obtidos noutros estudos mais recentes. Por exemplo, Utsumi e Mendes (2000) revelaram que à medida que o aluno passa do Ensino Fundamental para o Ensino Médio, a sua atitude face à matemática se torna mais negativa. Globalmente, os dados recolhidos sugeriam que os alunos com seis anos de escolaridade apresentavam uma atitude mais positiva face à matemática do que os seus colegas com sete e oito anos de escolaridade. Os estudantes sem retenções no seu percurso escolar apresentavam uma atitude consideravelmente mais positiva face à aprendizagem da matemática do que a apresentada pelos seus colegas que registavam pelo menos uma retenção no seu percurso de apren- dizagem. Em relação à idade, os alunos com 16 anos, ou mais, expressavam atitudes mais negativas face à matemática do que os alunos com idades entre os 11 e os 12 anos (cf., Watt, 2000; Utsumi \& Mendes, 2000).

Outro dos dados mais consistentes encontrados na literatura refere a diferença na atitude face à matemática em função do género (Núñez et al., 2005). Nos primeiros estudos realizados por Fennema e Sherman $(1977$; 1978) foram relatadas diferenças entre sexos relacionadas com o sucesso na matemática. Estas investigadoras analisaram a relação entre variáveis afectivas ou atitudinais e as crenças face a este domínio de aprendizagem, a percepção da utilidade dos conhecimentos matemáticos e a confiança dos alunos na sua competência para estas aprendizagens. Os seus dados sugerem que os homens mostram mais confiança face às tarefas escolares relacionadas com a matemática e acreditam que esta disciplina tem mais utilidade para eles do que para elas. Outros dados referem que os rapazes não sugerem qualquer estereótipo face à matemática como pertencendo a um "domínio masculino” (e.g., um pensamento do tipo: “matemática é coisa de homens", "para ter sucesso em matemática é preciso nascer homem”, etc.) (Fennema, 1993; Fennema \& Sherman, 1977, 1978). No entanto, as jovens participantes na pesquisa declararam que a matemática era mais apropriada para os homens que para as mulheres. Estes dados recolhidos na população jovem, a sua influência e o seu impacto diferencial nas mulheres e nos homens, foram confirmados posteriormente (e.g., Leder, 1992; Silva, 2005).

Thomas (2000), Willis (1995) e Fullarton (1993), entre outros autores, afirmam que esta atitude negativa das mulheres perante a aprendizagem da matemática contribui para o seu menor investimento e sucesso nas disciplinas que impliquem a mestria de conteúdos matemáticos. Mais recentemente, Schmader, Johns e Barquissau (2004) analisaram a variabilidade individual na representação dos estereótipos de género nas competências para a matemática, concluindo que uma percentagem significativa de mulheres assume a ideia de que os homens são superiores na matemática. Não obstante, nem todos os dados da literatura apontam a favor da hipótese da “matemática como um domínio masculino”. Por exemplo, Forgasz (2000) realizou uma revisão extensa para tentar contrastar a hipótese das supostas diferenças de género a favor dos homens na aprendizagem da matemática. Os seus dados, com estudantes australianos, revelaram que os rapazes consideravam a matemática mais difícil do que as raparigas, necessitando, inclusive, de ajuda adicional para superar os problemas propostos. Para além disso, as raparigas interessavam-se e apreciavam mais a matemática do que os rapazes. Estes resultados parecem, claramente, desafiar a hipótese da matemática como um domínio masculino. Neste mesmo sentido, Kloosterman et al. (2001) realizaram uma pesquisa comparando as percepções de estudantes do Secundário e da Universidade sobre a relação entre género, aprendizagem e rendimento na matemática. Estes autores concluíram que os grupos de estudantes, de zonas rurais e urbanas, acreditavam que, em geral, o desempenho na matemática não se resu- 
mia a uma questão de género. As mulheres defendiam, inclusivamente, esta posição com mais firmeza do que os homens. Estes autores concluíram que os alunos e alunas gostam identicamente da matemática, não se diferenciando relativamente à sua crença no que concerne ao grau de dificuldade percebida e à importância desta disciplina para a sua vida futura.

Hanna (2003) apresenta um estudo de revisão da literatura face aos resultados de equidade na matemática desde a década de sessenta. Para clarificar as diferenças de género relativamente ao sucesso face à matemática, foram analisados três estudos da International Association for the Evaluation of Educational Achievement (IEA): First, Second, and Third International Mathematics Science Study, (FIMS, SIMS e TIMSS, respectivamente). Comparando os resultados dos três estudos do IEA, verificou-se que o impacto das diferenças de género no sucesso escolar varia de um país para o outro. Tendo em consideração os resultados dos estudos revistos, ao contrário do que acontecia há 30 ou 40 anos atrás, actualmente não existem dados conclusivos sobre as diferenças de género relativamente à percepção de competência, ao grau de domínio dos conhecimentos nesta área académica ou às atitudes face à aprendizagem da matemática. Os dados variam de estudo para estudo dependendo das variáveis consideradas nas pesquisas. Algumas das mais importantes parecem ser o tipo de escola (se é mista ou não), a cultura do país, o grau de ensino considerado e a idade dos estudantes.

No presente trabalho, que encaramos como um pequeno subsídio para esta área de investigação, analisamos apenas dados relativos às diferenças de género tendo em consideração o ano de escolaridade e o contexto educativo, nomeadamente, a escolaridade obrigatória do sistema educativo espanhol.

\section{Método}

\section{Participantes}

A amostra tomada foi constituída por alunos de escolas públicas da província das Astúrias, em Espanha, da Educação Secundária Obrigatória (ESO) - etapa correspondente ao percurso escolar, no ensino brasileiro, entre a $7^{\underline{a}}$ e a $10^{\underline{a}}$ séries (cf. Tabela 1). A amostra foi obtida mediante um procedimento intencional, utilizando o sexo e o ano de escolaridade como variáveis indicadoras. A amostra total foi composta por 2672 estudantes dos níveis de ensino já mencionados, dos quais 1328 são mulheres e 1344 homens com idades compreendidas entre os 12 e os 16 anos. A Tabela 1 apresenta a distribuição dos alunos pelos diferentes subgrupos analisados.

\section{Instrumentos}

Nesta pesquisa foi utilizado o Inventário de Atitudes Face à Matemática (IAM). Trata-se de uma versão ampliada com modificações significativas da escala de avaliação de atitudes face à matemática (FSS), elaborada por Fennema e Sherman (1978) e utilizada desde então por inúmeros investigadores. O IAM é constituído por grande parte dos itens do FSS (adaptados à cultura espanhola), assim como por novos itens destinados a medir o tipo de orientação motivacional associado à atitude e ao tipo de atribuição causal realizada (necessária para compreender correctamente a atitude prevalente). No total, o IAM possui 86 itens que avaliam 15 dimensões: falta de confiança no sucesso futuro, pensamento estereotipado, competência percebida, utilidade percebida, motivação intrínseca, motivação para o sucesso, interesse por evitar o envolvimento, ausência de interesse na matemática, ansiedade, sentimentos-emoções, atribuição do sucesso à capacidade, atribuição do sucesso a causas externas (e.g., ser o preferido do professor), atribuição do insucesso a causas externas (e.g., falta de competência dos professores), atitude percebida dos pais e atitude percebida dos professores. A análise factorial realizada confirmou a estrutura teórica mencionada de 15 factores, explicando 60,7 \% da variância. A fiabilidade das subescalas é bastante aceitável, oscilando entre uma consistência interna moderada de 0,60 , relativa aos itens do factor 14 (atitude dos professores), o único valor abaixo do recomendado, e uma elevada consistência interna de 0,88 relativa ao factor 1 (incapacidade percebida para a matemática) (González-Pienda et al., 2002; Silva, 2005).

\section{Procedimentos}

Para a contrastação do objectivo apresentado neste estudo foram realizadas diferentes análises multivariadas da

Tabela 1

Distribuição dos alunos em função do sexo e do ano de escolaridade

\begin{tabular}{cccccc}
\hline \multirow{2}{*}{ Sexo } & \multicolumn{4}{c}{ Ano de Escolaridade } & \multirow{2}{*}{ Total } \\
\cline { 2 - 5 } & $1^{\underline{0}}$ ESO & $2^{\underline{0}}$ ESO & $3^{\underline{0}}$ ESO & $4^{\underline{0}}$ ESO & \\
\cline { 2 - 5 } & $n(\%)$ & $n(\%)$ & $n(\%)$ & $n(\%)$ & $N(\%)$ \\
\hline Mulheres & $263(19,8)$ & $257(19,4)$ & $402(30,3)$ & $406(30,6)$ & $1328(100)$ \\
Homens & $245(18,2)$ & $293(21,8)$ & $447(33,3)$ & $359(26,7)$ & $1344(100)$ \\
\hline
\end{tabular}

ESO = Educação Secundária Obrigatória 
variância (MANOVA), utilizando como variáveis independentes o género e o ano de escolaridade e, variáveis dependentes, as 15 dimensões do IAM.

\section{Resultados}

Na Tabela 2 é apresentada a estatística descritiva das variáveis analisadas nesta pesquisa (tamanho da amostra, médias e desvios-padrão).

\section{Efeitos conjuntos}

Os resultados das análises multivariadas indicam que, tanto a variável género, como a variável ano de escolaridade explicam, significativamente, a variabilidade observada na totalidade das variáveis dependentes analisadas (correspondentes às dimensões do Inventário das Atitudes face à Matemática, IAM) (valor de Lamda de Wilks $=0,905 ; F_{15,1252}=$ 8,806; $p<0,001 ; \eta^{2}=0,0095$, e valor de Lamda de Wilks = 0,$828 ; F_{45,3720}=5,410 ; p<0,001 ; \eta^{2}=0,061$, respectivamente). A interacção entre o género e o ano não é estatisticamente significativa (valor de Lamda de Wilks $=0,960 ; F_{45,3720}=1,155$; $\left.p=0,222 ; \eta^{2}=0,014\right)$.

As provas dos efeitos inter-sujeitos para cada uma das variáveis dependentes (dimensões da escala IAM), indicam que as diferenças globais encontradas se devem ao efeito do género e da variável covariada ano de escolaridade sobre a totalidade das variáveis dependentes, salvo a dimensão "sentimentos-emoções relacionados com a aprendiza- gem da matemática”. Mais concretamente, obtêm-se efeitos estatisticamente significativos em relação a: falta de confiança (expectativas face ao fracasso na matemática) $\left(F_{7}, 1266\right.$ $\left.=6,615 ; p<0,001 ; \eta^{2}=0,035\right)$; pensamento estereotipado $\left(F_{7}\right.$ , $\left.{ }_{1266}=11,163 ; p<0,001 ; \eta^{2}=0,058\right)$; grau de utilidade percebida da matemática no futuro $\left(F_{7,1266}=11,810 ; p<0,001 ; \eta^{2}\right.$ $=0,061)$; falta de interesse pela matemática $\left(F_{7,1266}=5,029\right.$; $\left.p<0,001 ; \eta^{2}=0,027\right)$; competência percebida para a matemática $\left(F_{7,1266}=15,103 ; p<0,001 ; \eta^{2}=0,077\right)$; motivação para o sucesso (interesse em sobressair na matemática) $\left(F_{7,1266}=\right.$ 11,805; $\left.p<0,001 ; \eta^{2}=0,061\right)$; atitude percebida dos pais em relação à importância da matemática $\left(F_{7,1266}=6,193 ; p<\right.$ 0,$\left.001 ; \eta^{2}=0,033\right)$; ansiedade face às tarefas de matemática $\left(F_{7,1266}=10,917 ; p<0,001 ; \eta^{2}=0,057\right)$; interesse em ocultar a competência na matemática $\left(F_{7,1266}=4,016 ; p<0,001 ; \eta^{2}=\right.$ 0,022); atribuição do sucesso a causas externas (como por exemplo, ser o favorito do professor) $\left(F_{7,1266}=5,769 ; p<\right.$ 0,$\left.001 ; \eta^{2}=0,031\right)$; atribuição do sucesso à capacidade $\left(F_{7}\right.$ $\left.{ }_{1266}=4,152 ; p<0,001 ; \eta^{2}=0,022\right)$; atribuição do fracasso a causas externas (como, por exemplo, a incompetência do professor) $\left(F_{7,1266}=7,666 ; p<0,001 ; \eta^{2}=0,041\right)$ e a atitude dos professores em relação à competência do estudante para enfrentar com sucesso as tarefas de matemática $\left(F_{7}, 1266\right.$ $\left.=10,792 ; p<0,001 ; \eta^{2}=0,056\right)$. Como se afirmou anteriormente, não foram encontradas diferenças estatisticamente significativas no que concerne à dimensão sentimentosemoções provocados pela matemática $\left(F_{7,1266}=1,066 ; p=\right.$ 0,$\left.383 ; \eta^{2}=0,006\right)$.

Tabela 2

Médias, desvios-padrão e tamanho das amostras em função do género e do ano de escolaridade

\begin{tabular}{|c|c|c|c|c|c|c|c|c|c|c|c|c|c|c|c|c|}
\hline \multirow{3}{*}{ Dimensões do IAM } & \multicolumn{4}{|c|}{$1^{\mathbf{0}} \mathrm{ESO}$} & \multicolumn{4}{|c|}{$2^{\underline{\mathbf{0}}} \mathrm{ESO}$} & \multicolumn{4}{|c|}{ 3ํㅡ ESO } & \multicolumn{4}{|c|}{$4^{0} \mathrm{ESO}$} \\
\hline & \multicolumn{2}{|c|}{$\begin{array}{l}\text { Mulheres } \\
(n=263)\end{array}$} & \multicolumn{2}{|c|}{$\begin{array}{l}\text { Homens } \\
(n=245)\end{array}$} & \multicolumn{2}{|c|}{$\begin{array}{l}\text { Mulheres } \\
(n=257)\end{array}$} & \multicolumn{2}{|c|}{$\begin{array}{l}\text { Homens } \\
(n=293)\end{array}$} & \multicolumn{2}{|c|}{$\begin{array}{l}\text { Mulheres } \\
(n=402)\end{array}$} & \multicolumn{2}{|c|}{$\begin{array}{l}\text { Homens } \\
(n=447)\end{array}$} & \multicolumn{2}{|c|}{$\begin{array}{l}\text { Mulheres } \\
(n=406)\end{array}$} & \multicolumn{2}{|c|}{$\begin{array}{l}\text { Homens } \\
(n=359)\end{array}$} \\
\hline & $M$ & $D P$ & $M$ & $D P$ & $M$ & $D P$ & $M$ & $D P$ & $M$ & $D P$ & $M$ & $D P$ & $M$ & $D P$ & $M$ & DP \\
\hline Falta de confiança & 2,49 & 1,21 & 2,36 & 1,12 & 2,64 & 1,15 & 2,47 & 1,09 & 2,92 & 1,12 & 2,55 & 1,14 & 2,99 & 1,17 & 2,81 & 1,17 \\
\hline Pensamento estereotipado & 1,75 & 0,93 & 1,92 & 1,06 & 1,66 & 0,85 & 2,12 & 1,04 & 1,59 & 0,90 & 2,14 & 1,11 & 1,48 & 0,84 & 1,95 & 0,94 \\
\hline Utilidade da matemática para o futuro & 4,03 & 0,88 & 4,00 & 1,03 & 3,85 & 0,92 & 3,94 & 0,82 & 3,54 & 0,97 & 3,57 & 1,02 & 3,42 & 0,97 & 3,40 & 0,93 \\
\hline Falta de interesse pela matemática & 4,06 & 0,97 & 4,04 & 1,17 & 3,86 & 0,95 & 3,91 & 1,01 & 3,73 & 1,00 & 3,59 & 1,03 & 3,75 & 0,92 & 3,60 & 0,94 \\
\hline Competência percebida na matemática & 3,76 & 0,84 & 3,87 & 0,85 & 3,71 & 0,80 & 3,85 & 0,79 & 3,36 & 0,90 & 3,67 & 0,96 & 3,17 & 0,82 & 3,41 & 0,83 \\
\hline Motivação para o sucesso & 3,93 & 0,96 & 3,94 & 1,04 & 3,75 & 0,98 & 3,98 & 0,81 & 3,69 & 0,92 & 3,57 & 1,10 & 3,54 & 1,00 & 3,53 & 0,97 \\
\hline Motivação intrínseca & 3,28 & 0,97 & 3,48 & 0,97 & 3,37 & 0,87 & 2,93 & 0,87 & 3,31 & 0,90 & 3,12 & 0,91 & 2,84 & 0,88 & 3,11 & 1,00 \\
\hline Atitude dos pais face à matemática & 4,26 & 0,81 & 4,23 & 0,89 & 4,13 & 0,88 & 4,13 & 0,79 & 3,99 & 0,94 & 3,95 & 0,84 & 3,90 & 0,86 & 3,74 & 0,87 \\
\hline Ansiedade ante a matemática & 3,06 & 1,01 & 3,40 & 1,13 & 3,01 & 1,00 & 3,39 & 1,04 & 2,70 & 1,05 & 3,28 & 1,04 & 2,78 & 0,98 & 2,97 & 1,03 \\
\hline Interesse por ser o melhor em matemática & 2,10 & 1,00 & 2,18 & 1,12 & 1,86 & 0,94 & 2,14 & 1,16 & 1,90 & 0,98 & 2,13 & 1,14 & 1,74 & 0,95 & 2,07 & 1,05 \\
\hline $\begin{array}{l}\text { Atribuição do êxito a ser o(a) favorito(a) } \\
\text { do professor }\end{array}$ & 1,83 & 0,94 & 2,16 & 1,18 & 1,94 & 0,96 & 2,45 & 1,11 & 2,05 & 0,99 & 2,37 & 1,08 & 1,93 & 0,88 & 2,33 & 1,01 \\
\hline Atribuição do êxito-fracasso à capacidade & 2,56 & 1,20 & 2,85 & 1,11 & 2,69 & 1,09 & 2,85 & 1,09 & 2,68 & 1,10 & 3,11 & 1,10 & 2,71 & 1,02 & 2,96 & 1,00 \\
\hline $\begin{array}{l}\text { Atribuição do fracasso à incompetência do } \\
\text { professor }\end{array}$ & 2,20 & 1,22 & 2,35 & 1,22 & 2,27 & 1,09 & 2,50 & 1,19 & 2,82 & 1,05 & 2,85 & 1,14 & 2,57 & 0,90 & 2,63 & 0,94 \\
\hline $\begin{array}{l}\text { Sentimentos-emoções provocados pela } \\
\text { matemática }\end{array}$ & 2,33 & 1,00 & 2,31 & 1,08 & 2,47 & 0,99 & 2,52 & 1,09 & 2,50 & 0,93 & 2,52 & 1,04 & 2,47 & 0,91 & 2,53 & 0,91 \\
\hline $\begin{array}{l}\text { Atitudes dos professores face às possibilida- } \\
\text { des do(a) aluno(a) na matemática }\end{array}$ & 3,75 & 1,00 & 3,84 & 1,00 & 3,60 & 0,90 & 3,76 & 0,96 & 3,29 & 0,92 & 3,43 & 0,90 & 3,23 & 0,88 & 3,33 & 0,79 \\
\hline
\end{tabular}

IAM = Inventario de Atitudes face à Matemática. ESO = Educação Secundária Obrigatória. $M=$ média; $D P=$ desvio padrão 


\section{Ano de escolaridade e atitude face à matemática}

A influência desta variável é estatisticamente significativa face às seguintes dimensões: falta de confiança (expectativas de fracasso na matemática) $\left(F_{3,1273}=8,225 ; p<0,001\right.$; $\left.\eta^{2}=0,019\right)$; utilidade da matemática para o futuro $\left(F_{3,1273}=\right.$ 26,994; $\left.p<0,001 ; \eta^{2}=0,060\right)$; falta de interesse pela matemática $\left(F_{3,1273}=10,690 ; p<0,001 ; \eta^{2}=0,025\right)$; competência percebida para matemática $\left(F_{3,1273}=25,858 ; p<0,001 ; \eta^{2}=0,058\right)$; motivação de sucesso (interesse por sobressair na matemática) $\left(F_{3,1273}=10,973 ; p<0,001 ; \eta^{2}=0,025\right)$; motivação intrínseca $\left(F_{3,1273}=18,452 ; p<0,001 ; \eta^{2}=0,042\right)$; atitude dos pais face à importância da matemática $\left(F_{3,1273}=13,921 ; p<0,001 ; \eta^{2}=\right.$ $0,032)$; ansiedade ante a matemática $\left(F_{3,1273}=8,457 ; p<0,001\right.$; $\left.\eta^{2}=0,020\right)$; interesse em ocultar a (in)competência na matemática $\left(F_{3,1273}=2,642 ; p=0,048 ; \eta^{2}=0,006\right)$; atribuição do êxito a causas externas (como por exemplo, ser o favorito do professor) $\left(F_{3,1273}=2,828 ; p=0,037 ; \eta^{2}=0,007\right)$; atribuição do fracasso a causas externas (como, por exemplo, a incompetência do professor) $\left(F_{3,1273}=16,178 ; p<0,001 ; \eta^{2}=0,037\right)$ e, finalmente, a atitude dos professores face à competência do aluno para enfrentar com sucesso as tarefas de matemática $\left(F_{3,1273}=22,255 ; p<0,001 ; \eta^{2}=0,050\right)$.

Pelo contrário, não são estatisticamente significativas as diferenças obtidas para as três restantes dimensões: pensamento estereotipado $\left(F_{3,1273}=2,251 ; p=0,081 ; \eta^{2}=0,005\right)$; atribuição do sucesso à capacidade $\left(F_{3,1273}=1,756 ; p=0,154\right.$; $\left.\eta^{2}=0,004\right)$ e sentimentos-emoções provocados pela matemática $\left(F_{3,1273}=2,347 ; p=0,071 ; \eta^{2}=0,050\right)$.

\section{Género e atitude face à matemática}

A variável género, por sua vez, explica de modo significativo nove das quinze dimensões do IAM. Foram encontradas diferenças estatisticamente significativas entre homens e mulheres nas seguintes dimensões: falta de confiança (expectativas de fracasso na matemática) $\left(F_{1,1274}=13,844\right.$; $\left.p<0,001 ; \eta^{2}=0,011\right)$; pensamento estereotipado $\left(F_{1,1274}=\right.$ 57,320; $\left.p<0,001 ; \eta^{2}=0,043\right)$; competências percebidas para a matemática $\left(F_{1,1274}=16,246 ; p<0,001 ; \eta^{2}=0,013\right)$; motivação intrínseca $\left(F_{1,1274}=22,135 ; p<0,001 ; \eta^{2}=0,017\right)$; ansiedade face à matemática $\left(F_{1,1274}=39,355 ; p<0,001 ; \eta^{2}=0,030\right)$; interesse em ocultar a competência na matemática $\left(F_{1,1274}=\right.$ 14,467; $\left.p<0,001 ; \eta^{2}=0,011\right)$; atribuição do sucesso a causas externas (como por exemplo, ser o preferido do professor) $\left(F_{1,1274}=29,441 ; p<0,001 ; \eta^{2}=0,023\right)$; atribuição do sucesso à capacidade $\left(F_{1,1274}=20,734 ; p<0,001 ; \eta^{2}=0,016\right)$ e, finalmente, atitude dos professores em relação à competência do estudante para enfrentar com sucesso as tarefas de matemática $\left(F_{1,1274}=5,492 ; p=0,019 ; \eta^{2}=0,004\right)$.

Por outro lado, as diferenças obtidas não são estatisticamente significativas para as seguintes dimensões: utilidade da matemática para o futuro $\left(F_{1,1274}=0,187 ; p=0,665 ; \eta^{2}=\right.$ $0,000)$; falta de interesse pela matemática $\left(F_{1,1274}=1,709 ; p=\right.$ 0,$\left.191 ; \eta^{2}=0,001\right)$; motivação de sucesso (interesse por sobressair na matemática) $\left(F_{1,1274}=0,107 ; p=0,744 ; \eta^{2}=0,000\right)$; atitude dos pais face à importância da matemática $\left(F_{1,1274}=\right.$ 1,326; $\left.p=0,250 ; \eta^{2}=0,001\right)$; atribuição do fracasso a causas externas (como a incompetência do professor) $\left(F_{1,1274}=3,681\right.$; $\left.p=0,055 ; \eta^{2}=0,003\right)$ e sentimentos-emoções provocados pela matemática $\left(F_{1,1274}=0,217 ; p=0,642 ; \eta^{2}=0,000\right)$.

\section{Discussão}

Os resultados obtidos mostram um efeito estatisticamente significativo da variável género sobre as diferentes dimensões do IAM. Contudo, também sugerem que tais efeitos se encontram, poderosamente, mediados pela variável ano de escolaridade. Desta forma, é possível afirmar que com o avanço na escolaridade (do primeiro ao último ano da Educação Secundária Obrigatória), se observa um decréscimo das expectativas de sucesso futuro, do pensamento estereotipado e da utilidade da matemática no futuro. Seguidamente, abordaremos detalhadamente estes e outros resultados da nossa pesquisa.

Em primeiro lugar, é relevante que o interesse pela matemática decresça significativamente à medida que se ascende nos anos de escolaridade (embora sempre ligeiramente mais baixo nos rapazes), resultado que é, no mínimo surpreendente, se se considerar a relevância do conhecimento matemático face às oportunidades futuras de ordem académica, já que os estudos superiores, cuja saída profissional é mais procurada pela sociedade actual, requerem competência nesta matéria (Koehler \& Grouws, 1992).

Em segundo lugar, e relacionado com o exposto anteriormente, também se observou o facto de a utilidade da matemática face ao futuro apresentar um decréscimo significativo mais acentuado à medida que se avança no ano de escolaridade. Este facto talvez se explique pela forma como, na maioria das vezes, se explica e apresenta a matemática de forma descontextualizada e alheada da vida real, de modo que os estudantes não percebem qual a relação dos conteúdos matemáticos estudados com a melhoria da sua competência para resolver problemas da vida quotidiana (Utsumi \& Mendes, 2000). Daí, a insistência de muitos investigadores, na necessidade de relacionar a teoria com a prática, especialmente no ensino de alunos menos competentes ou daqueles que apresentam um ritmo de aprendizagem mais lento (Erickan, McCreith, \& Lapointe, 2005; Furner, Yahya, \& Duffy, 2005; González-Pienda \& Alvarez, 1998).

Em terceiro lugar, observa-se que a competência percebida para a aprendizagem e sucesso na matemática diminui significativamente à medida que os estudantes vão avançando do ensino primário para os últimos anos do ensino secundário. Este facto talvez se encontre relacionado com o comportamento dos docentes, já que os estudantes se apercebem das expectativas que os professores constroem sobre o seu sucesso futuro e confirmam com o seu rendimento as expectativas dos professores (Núñez et al., 1998, 2005). Quando participam nas tarefas de matemática, os alunos não só aprendem conteúdos matemáticos, como também recolhem dados sobre a sua capacidade para a matemática. Esta última aprendizagem é muito importante já que constitui o fundamento da competência percebida. A investigação realizada 
no campo da aprendizagem escolar em geral, e da aprendizagem auto-regulada em particular, indica claramente a importância das crenças de auto-eficácia e do controlo percebido como aspectos críticos para a motivação e persistência inicial, mas também como factor protector face às dificuldades e aos primeiros fracassos (e.g., Gaskill \& Woolfolk, 2002; Pajares \& Graham, 1999; Pintrich, 2003; Valle et al., 2003; Zimmerman, 2002).

Em quarto lugar, uma vez perdida a confiança na capacidade própria, esta afecta, directamente, o próprio interesse pela matéria, e desperta sentimentos e emoções negativas, aumentando significativamente a ansiedade (Rosário \& Soares, 2004). Curiosamente, no início da escolaridade, a ansiedade não está presente nas actividades escolares; é a partir do segundo ciclo do ensino primário que os níveis de ansiedade aumentam consideravelmente. A associação encontrada entre os níveis de ansiedade e os níveis de interesse pelo sucesso na matemática pode indicar que existe uma significativa preocupação com a descida do rendimento nesta área (Rosário, Soares, Núñez, González-Pienda \& Simões, 2004). A redução dos níveis de ansiedade e dos sentimentos de culpabilidade pode resultar de dois caminhos: (a) a melhoria do desempenho na matemática, ou (b) o uso de estratégias defensivas (por exemplo, reduzindo o interesse pela matemática e baixando a sua utilidade percebida face ao futuro; Rodríguez, González-Cabanach, Valle, Núñez, \& González-Pienda, 2004). Este segundo caminho parece ser o escolhido pelos alunos. No Secundário os dados mostram um decréscimo significativo da ansiedade e dos sentimentos negativos, a par de uma recuperação da auto-estima, isto, apesar da trajectória descendente no rendimento em matemática.

Os dados aportados pela nossa pesquisa indicam que não é possível estimar o efeito do género sobre as atitudes face à matemática, sem ter em consideração o efeito de outras variáveis significativas como seja, neste caso, o ano de escolaridade, mas também a organização curricular e o capital cultural da população investigada. Estas variáveis de natureza institucional e cultural podem ser, até mesmo, uma parte substantiva da explicação para as discrepâncias dos dados apresentados nas investigações referenciadas na literatura (cf. Ercikan, McCreith, \& Lapointe, 2005; Fennema, 1993; Hanna, 2003; Thomas, 2000). Trabalhos futuros deveriam considerar o papel explicativo destas dimensões na atitude e no sucesso escolar na matemática (Núñez et al., 2005).

Atendendo aos efeitos observados, os dados desta pesquisa sugerem, por exemplo, que as mulheres, por contraponto aos homens, apresentam: (1) mais falta de confiança nos seus sucessos futuros na área da matemática; (2) um pensamento mais estereotipado (e.g., sustentando mais vezes que a "matemática é coisa de homens”); (3) menor competência percebida para a aprendizagem da matemática; (4) menor ansiedade face à matemática; e (5) menor atribuição do sucesso a causas externas (e.g., ser o favorito do professor). Os homens apresentam um maior interesse por evitar "parecer bons a matemática”, acreditando, em maior grau, que o sucesso nesta matéria se deve à capacidade. Estes resultados, evi- denciando claros efeitos de género, deveriam ser tidos em consideração, por exemplo, nos projectos de intervenção em educação matemática desenvolvidos com rapazes da classe baixa de modo a promover o seu sucesso escolar, evitando o abandono da escolaridade obrigatória.

Estes dados, replicando outros de pesquisas já referenciadas, constituem mais um motivo para todos - universidade, docentes do ensino fundamental e médio, encarregados de educação, políticos -, pensarmos as atitudes face à matemática, e repensarmos as nossas práticas a fim de promovermos o sucesso instrutivo e educativo, também neste domínio académico.

\section{Referências}

Bishop, A. (2000). Enseñanza de las matemáticas: ¿como beneficiar a todos los alumnos? In N. Gregório, A. Deulofeu, \& A. Bishop (Orgs.), Matemática y Educación (pp. 35-56). Barcelona: Graó.

Erickan, K., McCreith, T., \& Lapointe, V. (2005). Factors associated with mathematics achievement and participation in advanced mathematics courses: an examination of gender differences from an international perspective. School Science and Mathematics, 105(1), 5-10.

Fennema, E. (1993). Gender equity for mathematics and science. Comunicação apresentada em colóquio acadêmico, University of Wisconsin, Madison.

Fennema, E., \& Sherman, J. A. (1977). Sex-related differences in mathematics achievement, spatial visualization, and affective factors. American Educational Research Journal, 14(1), 51-71.

Fennema, E., \& Sherman, J. A. (1978). Sex-related differences in mathematics achievement, and related factors: a further study. Journal for Research in Mathematics Education, 9(3), 189-203.

Forgasz, H. J. (2000). The gender-stereotyping of mathematics: pre-service teachers'views. Comunicação apresentada na conferência anual da Australian Association for Research in Education, Sydney.

Fullarton, S. (1993). Confidence in mathematics: the effects of gender. Geelong, Victoria, Australia: Deakin University Press.

Furner, J. M., Yahya, N., \& Duffy, M. L. (2005). Teach Mathematics: strategies to reach all students. Intervention in School and Clinic, 41(1), 16-23.

Gaskill, P. J., \& Woolfolk, A. E. (2002). Self-efficacy and self-regulated learning: the dynamic duo in school performance. In J. Aronson (Org.), Improving academia achievement: impact of psychological factors on education (pp. 185-206). San Diego, California: Academic.

González-Pienda, J. A., \& Alvarez, L. (1998). Dificultades específicas relacionadas con el aprendizaje de las matemáticas. In J. A. González-Pienda \& J.C. Núñez (Orgs.), Dificultades de aprendizaje escolar (pp. 315-340). Madri: Pirámide.

González-Pienda, J. A., Núñez, J. C., Alvarez, L., González, P., Soler, E., González-Pumariega, S., \& Roces, C. (2002). Aplicación de la estrategia hypertexto para la enseñanza y el aprendizaje de las matemáticas en la educación secundaria. In J. N. García (Org.), Aplicaciones de intervención psicopedagógica (pp. 215-228). Madri: Pirámide.

Hanna, G. (2003). Reaching gender equity in Mathematics education. The Educational Forum, 67(3), 204-214.

Kloosterman, P., Tassel, J. H., Ponniah, A. G., \& Essex, N. K. (2001). Mathematics as a genderer domain in the United States. The American Educational Research Association, 13, 1-15.

Koehler, M., \& Grouws, D. A. (1992). Mathematics teaching and practices and their effects. In D. A. Grouws (Org.), Handbook of research on Mathematics teaching and learning (pp. 115-126). Nova York: MacMillan.

Leder, G. C. (1992). Mathematics and gender: changing perspectives. In D. A. Grouws (Org.), Handbook of research on Mathematics teaching and learning (pp. 597-622). Nova York: MacMillan. 
McLeod, D. B. (1992). Research on affect in mathematics education. A reconceptualization. In D. A. Grouws (Org.), Handbook of research on Mathematics teaching and learning (pp. 575-596). Nova York: MacMillan.

Núñez, J. C., González-Pienda, J. A., Alvarez, L., González-Castro, P., GonzálezPumariega, S., Roces, C., Castejón, L., Bernardo, A., Solano, P., García, D., Da Silva, E. H, Rosário, P., \& Rodrigues, L. S. (2005). Las actitudes hacia las matemáticas: perspectiva evolutiva. In Actas do VIII Congreso Galaico-Portugués de Psicopedagoxía (pp. 2389-2396). Braga: Universidade do Minho; Universidade da Corunha.

Núñez, J. C., González-Pienda, J. A., Rodríguez, M., González-Pumariega, S., Roces, C., Alvarez, L., \& González-Torres, M. C. (1998). Estrategias de aprendizaje, autoconcepto y rendimiento académico. Psicothema, 10, 97-109.

Pajares, F., \& Graham, L. (1999). Self-efficacy, motivation constructs, and mathematics performance of entering middle school students. Contemporary Educational Psychology, 24, 124-139.

Pintrich, P. R. (2003). Motivation and classroom learning. In W. M. Reynolds \& G. E. Miller (Orgs.), Handbook of Psychology: Educational Psychology (vol. 7, pp. 103-122). Hoboken, New Jersey: Wiley.

Rodríguez, S., González-Cabanach, R., Valle, A., Núñez, J. C., \& GonzálezPienda, J. A. (2004). Diferencias en el uso del self-handicapping y pesimismo defensivo y su relación con las metas de logro, la autoestima y las estrategias de autorregulación del aprendizaje. Psicothema, 16(4), 625-631.

Rosário, P., \& Soares, S. (2004). Questionário de Ansiedade face aos Testes (Q.A.T). In M. M. Gonçalves, M. R. Simões, L. S. Almeida, \& C.
Machado (Orgs.), Avaliação Psicológica. Instrumentos validados para a população portuguesa (Vol. II, pp. 39-51). Coimbra: Quarteto.

Rosário, P., Soares, S., Núnez, J. C., González-Pienda, J., \& Simões, F. (2004) Ansiedade face aos testes e auto-regulação da aprendizagem: variáveis emocionais no aprender. Psicologia e Educação, 3(1), 15-26.

Schmader, T., Johns, M., \& Barquissau, M. (2004). The costs of accepting gender differences: the role of stereotype endorsement in women's experience in the math domain. Sex Roles, 50(11-12), 835-850.

Silva, E. (2005). Actitudes ante las matemáticas. Tese de doutorado não-publicada, Departamento de Psicologia, Universidade de Oviedo, Espanha.

Thomas, J. P. (2000). Influences on mathematics learning and attitudes among African American high school students. The Journal of Negro Education, 69(3), 165-183.

Utsumi, M. C., \& Mendes, C. R. (2000). Researching the attitudes towards mathematics in basic education. Educational Psychology, 20(2), 237-243.

Valle, A., González-Cabanach, R., Núñez, J. C., González-Pienda, J. A., Rodríguez, S., \& Piñeiro, I. (2003). Multiple goals, motivation, and academic learning. British Journal of Educational Psychology, 73, 71-87.

Watt, G. (2000). Measuring attitudinal change in mathematics and English over $1^{\text {st }}$ year of junior high school: a multidimensional analysis. The Journal of Experimental Education, 68(4), 331-361.

Willis, S. (1995). Gender justice and the mathematics curriculum: four perspectives. In I. Parker, L. Rennie, \& B. Fraser (Orgs.), Gender, science and mathematics; shortening the shadow (pp. 41-51). Dordecht: Kluwer.

Zimmerman, B. J. (2002). Becoming self-regulated learner: an overview. Theory into Practice, 41, 64-72.

${ }^{1}$ Em Espanha, os resultados provêem de um trabalho de investigação financiado com fundos do projecto B.S.O.2002-01295 do Ministério da Ciência e Tecnologia (MCYT). Em Portugal, os trabalhos foram realizados com o subsídio de investigação da empresa F. Ramada, Aços e Indústrias, S.A.

Júlio A. Gonzalez-Pienda, doutor em Psicologia da Educação, é professor de graduação e pós-graduação em Psicologia na Universidade de Oviedo em Espanha.

José Carlos Núñez, doutor em Psicologia da Educação, é professor de graduação e pós-graduação em Psicologia na Universidade de Oviedo em Espanha.

Paula Solano, doutoranda em Psicología da Educação, é professora na Universidade de Oviedo, Espanha.

Elza Helena da Silva, doutora em Psicologia da Educação pela Universidade de Oviedo, é professora de graduação e pós-graduação na Universidade do Estado de Rio Grande do Norte, Brasil.

Pedro Rosário, doutor em Psicologia da Educação, é professor de graduação e pós-graduação em Psicologia na Universidade do Minho, Portugal.

Rosa Mourão, doutoranda em Psicologia da Educação no Departamento de Psicologia na Universidade do Minho, Portugal, é professora do ensino básico.

António Valle, doutor em Psicologia da Educação, é professor de graduação e pós-graduação em Psicologia na Universidade de A Corunha em Espanha.

Endereço para correspondência: Pedro Rosário; Departamento de Psicologia; Instituto de Educação e Psicologia; Universidade do Minho, Campus de Gualtar; 4710-049; Braga, Portugal. E-mail: prosario@iep.uminho.pt 